\title{
UNA LECTURA DE RÁFAGAS DE PÁJAROS DE RODRIGO EDUARDO GALARZA
}

Dora G. Villalba

\section{INTRODUCCIÓN. PRECISIONES SEMÁNTICAS}

Intentaré, en el transcurso de este escrito, una lectura "cooperativa" del libro del poeta correntino Rodrigo E. Galarza, (1) a quien conozco y con quien mantengo un diálogo fluido y permanente acerca de la labor poética.

Me aproximaré al texto desde la perspectiva simbólica. Para ello trataré de comunicar lo que entiendo por símbolo.

Según los griegos la palabra símbolo (sýmbolon) significa "nota, señal de reconocimiento. Contraseña militar, de hospedaje o teatral. "Indicio, contraseña, garantía, marca". (2) Surge de la combinación de "s y $n$ ", prefijo que denota la idea de "compañía, simultaneidad, igualdad, y el verbo "bállo", "tirar, arrojar, disparar, lanzar" y también figuradamente "imprimir en el ánimo, en la memoria”.(3)

Para precisar aún más acudiré a Jean CHEVALIER : " En su origen, el símbolo es un objeto cortado en dos trozos, sea de cerámica, madera o metal. Dos personas se quedan, cada una, con una parte, dos huéspedes, el acreedor y el deudor, dos peregrinos, dos seres que quieren separarse largo tiempo... Acercando las dos partes, reconocerán más tarde sus lazos de hospitalidad, sus deudas, su amistad. Los símbolos eran aún en la antigüedad signos de reconocimiento que permitian a los padres encontrar a sus hijos abandonados.[...] El simbolo deslinda y aúna: entraña las dos ideas de separación y de reunión: evoca una comunidad que ha estado divida y que debe reformarse. Todo símbolo implica una parte de signo roto; el sentido del símbolo se descubre en aquello que es a la vez rotura y ligazón de sus términos separados".(4)

A través de su etimologia y significado, por una parte, y del análisis de $J$. Chevalier, por otra, estoy en condiciones de puntualizar ciertos rasgos del símbolo:

a) por la presencia del prefijo "syn" alude a lo que está junto, lo que se ofrece a la vez, la idea es de convergencia.

b) el verbo "bállo" apunta a lo que se arroja.

De los dos elementos reunidos se deduce la significación de "poner juntamente, unir" y en su sentido figurado: "explicar, interpretar".

c) En lo expuesto por Chevalier encuentro una idea dominante: la división en dos de un objeto que luego se transpone a lo figurado como bipolaridad, y hasta como coincidentia oppositorum. 


\section{EL TITULO}

símbolo.

Por ahora comenzaré el recorrido de Ráfagas ... con la guía que enuncié sobre el

En este libro, Rodrigo me encamina a la elaboración de una hipótesis de lectura donde juegan elementos de la realidad cotidiana: "ráfagas ..." (movimiento violento de aire, golpe de luz instantáneo) y "pájaros" (nombre dado a las aves pequeñas, especialmente las del orden paseriforme). Lo interesante del título constituye la unión de dos componentes de la naturaleza como son las ráfagas; y los pájaros, pero indicando el segundo de los términos, la materia de que están hechas estas ráfagas. La relación sorprende y anima a seguir la interpretación. Creo que estas ráfagas de pájaros, "arrojadas juntamente" al lector lo movilizan a ver más allá de lo que aparece a simple vista como sencillo.

El aire, en la simbología, es uno de los cuatro elementos y se refiere a la espiritualización, al mundo intermedio entre el cielo y la tierra (asi como el poeta, para lós antiguos griegos, era una entidad intermedia entre dioses y hombres). También se asocia al aliento vital.Es lo sensible que simboliza lo invisible.

En cuanto a los pájaros, por su vuelo, se los asimila también a las relaciones entre el cielo y la tierra. Por su ligereza aluden a la liberación de la pesadez terrenal y, al mismo tiempo, al alma escapándose del cuerpo, "volando". Son lo opuesto a la serpiente como lo celeste se opone a lo terrestre y además, apuntan a los estados superiores del ser,

En esto reside la cualidad de lo simbólico: en pretender un acercamiento, hasta una coincidencia de significante y significado en una relación que nos aleje de la fría arbitrariedad que le adjudican los lingüistas al vocablo.

Puesta a interpretar el título (que probablemente confirmará la hipótesis de lectura) éste se me aparece como un Todo, donde el aire y los pájaros están indisolublemente integrados a lo superior, al espíritu, a esa zona "intermedia" entre el cielo y la tierra; zona a propósito para un poeta que, como hombre se acerca a los demás mortales pero, como poeta, es un demiurgo cuyo espíritu posee espacio donde se siente capaz de crear. Es cierto que no crea de la nada pero su creación surge de una combinación sorprendente de elementos, de una mirada diferente sobre los objetos y sus relaciones, porque se aparta de la rutinaria relación "lógica" de todos los dias, de lo que se ve a simple vista. Ahi reside la esencia y el meollo de la poesia. 


\section{EL CORPUS}

Más de veinte poemas constituyen el corpus de "Ráfagas ..." Todos ellos se incardinan a través de fuertes elementos simbólicos recurrentes. El hilo conductor son los pájaros. El símbolo-eje se encama en el pájaro-poeta.

\section{EL PÁJARO}

El mismo pájaro puede ser la inspiración del poeta y la imposibilidad de plasmarla con las palabras porque éstas no permiten expresarla en forma completa y acabada:

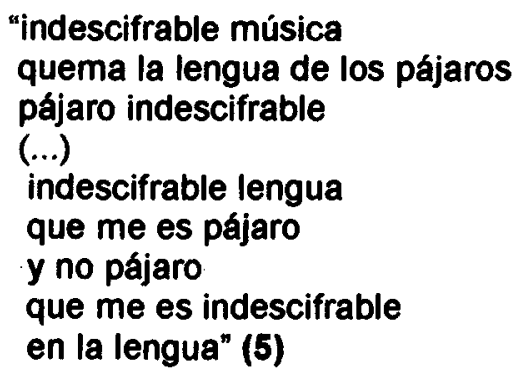

El pájaro es indescifrable en la lengua pues lo puramente verbal es incapaz de ofrecer a quien lee o escucha o piensa la totalidad en la unidad, pues el hablar o el escribir o el pensar están sometidos a la categoría temporal y adolecen de insoslayable linealidad.

O en un próximo paso, puede ser la palabra, que crea aún con sus límites e imperfecciones,

"la breve luz

o el leve pájaro

de la tardel

alguien sabrá

sentirla en sus manos"

"y vendrás

pájaro de mi silencio

a volarme la bocal

las llamas de polen

que encienden el horizonte

donde termina el desierto

y la luz

es la suave mirada del rocio

en la flor" (6) 
En el primer caso es el "leve pájaro" pues la inspiración del poeta es una especie de iluminación intensa y fugaz que no todos pueden traducir, en el preciso instante, en palabras. Por eso sólo "alguien sabrá sentir (esa breve luz o leve pájaro) en sus manos" y apoderarse de ella en un movimiento tan breve e intenso que le permita comunicar con el lenguaje (aunque imperfecto) esa vivencia única que es la inspiración.

Todo lo singular e irrepetible de ese movimiento que despierta la intuición está en el "pájaro de [...] silencio" que viene a "volar la boca" del poeta y viene también a fecundar con "llamas de polen que encienden el horizonte donde termina el desierto", la potencia creadora y a rescatarla de la aridez de lo consuetudinario, representado en la indeterminación estéril del desierto, para llegar a la luz evidenciada en el diálogo mudo entre rocío y flor, como motivo (en el sentido de movere) que echa a andar la poesía.

O puede ser el elemento determinante de la vida, sobre todo, en el siguiente poema que dice asi:

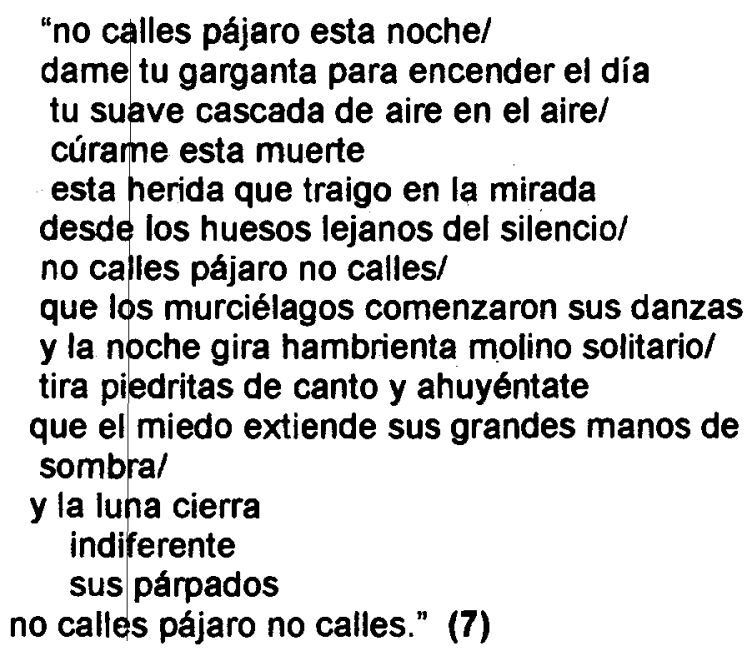

Se presenta un interesante contraste noche-día como figurador de las oposiciones

\section{CAOS - COSMOS O MUERTE-VIDA.}

El microcosmos-poeta es quien pide al pájaro-espíritu-inspiración-palabra que no lo abandone a la confusión de la no creación que pueda anonadario.

Así leemos: "dame tu garganta para encender el día" "cúrame esta muerte / esta herida que traigo en la mirada / desde los huesos lejanos del silencio". Este "no calles pájaro no calles" también lo rescata de la absoluta inmutabilidad de los 
elementos naturales ("la luna cierra indiferente [...]) frente a las posibilidades del creador cuando a éste no lo anima la suficiente emoción como para crear el mundo en cada palabra.

La presencia simbólica del pájaro se descubre también como : el vuelo espiritual que distingue al poeta como un ser diferente: "[...] sólo el pájaroll en su latido de vuelo// acrecienta el mundo" (8) o como el elemento esencial que permite al poeta elaborar el mundo con otra mirada y permanecer en él a pesar de la muerte, "tras la ráfaga temblarán mis pájaros// pero sus gargantas seguirán siendo el mundo." (9)

\section{OTROS SÍMBOLOS}

Sutilmente enlazados al símbolo fundamental aparecen otros elementos interpenetrados con él. De este modo se encuentra la LUZ, así nombrada, y también como llama, alba, día ...

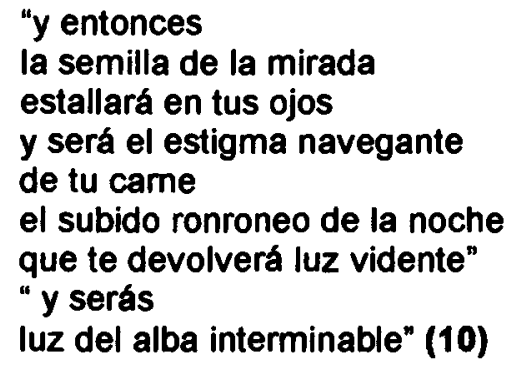

La luz en el sentido simbólico tiene connotaciones riquisimas. En los fragmentos anteriores aparece la luz vista en el sentido de la expansión creadora y también como esa chispa de luz divina que guarda en sí el poeta y que 10 acerca a Dios. Por este acercamiento, que no llega a igualarlo a la divinidad, es que todo poeta se ubica como una entidad intermedia entre la pura esencia de lo Superior y la llaneza del hombre común. $Y$ en esta lucha entre Espiritu y materia debe batallar con ambos extremos para hallar su naturaleza más alta y acercarla a lo divino y luminoso.

En el texto:

$$
\begin{aligned}
& \text { "y la luz } \\
& \text { es la suave mirada del rocio } \\
& \text { en la flor" (11) }
\end{aligned}
$$

La luz parte del poeta e ilumina el cosmos creativo.

En cambio en:

"un pájaro consume las últimas llamas de la tarde tarde de pájaros en llamas de últimos pájaros volando hacia el silencio". 
"y la flor

será una transparente llama" (12)

La luz se resuelve en llamas, esto es, en la luz como iluminación y ascenso del alma. Manifestación del fuego, la luz de la llama también figura la inmaterialidad del espiritu que busca la trascendencia. El mismo poeta se consume en esta llama que aspira a realizarse aunque después del esfuerzo creador, que se percibe como un instante sublime, se funda como la última luz de la tarde y llegue a su ocaso Impresiona como una parábola. Por eso aparece en contraste el símbolo opuesto a la luz: la noche, (por ejemplo, "el subido ronroneo de la noche") tomada aquí, creo, como momento de gestación, donde permanecen las posibilidades que cobrarán forma con la luz del día.

Unida también al símbolo eje: "PÁJARO", se presenta la LENGUA. El lenguaje, como invención humana es portador de serias limitaciones sobre todo, desde su perspectiva sistemática ordenada en la que, casi nada ya, queda por crear. Así se ofrece como un medio insuficiente para transmitir lo poético:

"indescifrable música

quema la lengua de los pájaros

[...]

indescifrable lengua

que me es pájaro

y no pájaro

que me es indescifrable

en la lengua"

"cuando el jadeo crece

en negra llamarada

y ya la lengua no cabe en la boca" (13)

Tal como Bécquer en la rima Nro. 11, Rodrigo Galarza se rebela contra las carencias del lenguaje para testimoniar los estados de la creación poética y la imposibilidad de no saber cómo decir lo que se quiere decir.

Al igual que Jano Bifronte y, como todo símbolo, el lenguaje se muestra en dos actitudes irreductibles: por una parte, parece prometer infinitas posibilidades de comunicación, pero, por otra, llegado a cierto punto, es impotente para transmitir lo único e inefable.

En un sorprendente juego ritmico también la MÚSICA es un símbolo frecuente en Ráfagas... Es ella la que inaugura el primer verso del primer poema: "Indescifrable música" y también "la música de la lengua", "la música de los pájaros", "otoño hendido de música". 
Me interesa destacar aquí que la música es otro elemento que permite que ef hombre se eleve del plano meramente humano y llegue a comunicarse con planos elevados en el sentido espiritual.

Además "es uno de los medios de asociarse a la plenitud de la vida cósmica"(14). Pero, ¿cómo juega la música en el mundo poético de Ráfagas ... ? Por todo lo dicho anteriormente se afianza como una posibilidad más para expresar lo inasible de la poesía. El poeta pretende, a través de ella, alcanzar el sentido pleno de sus vivencias y poder comunicarlas. Los límites de la condición humana, sin embargo, hacen ardua y difícil la tarea al punto que en algún momento, aparece esta música como indescifrable.

Todos los símbolos se subordinan para paladinar, en alguna medida, el dolor y el gozo del poeta al dar a luz su mensaje. Así lo testimonia él:

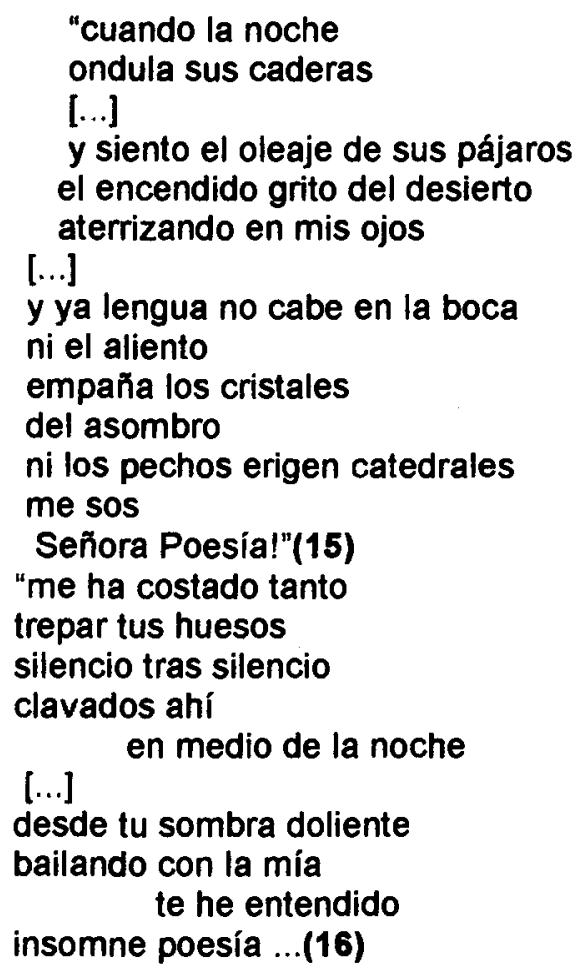

\section{CONCLUSIÓN}

Internarse en Ráfagas... es caminar por un universo simbólico riquísimo y pluridimensional.

Como el símbolo-objeto cortado en dos, Rodrigo Galarza "arroja" a su lector una serie de posibilidades que éste debe "completar" en un movimiento de lectura 
convergente con la escritura del autor. Así, con este movimiento cooperativo, puede uno internarse en el cosmos expresivo y co-crear desde la perspectiva simbólica del texto poético. Esto es factible pues el símbolo presenta diversas vertientes: explora la realidad con una mirada nueva; media entre lo prosaico de esa realidad y lo sublime de la visión del poeta; unifica con su fuerza elementos que parecen irreductibles; trasciende las fronteras de la pura racionalidad y el análisis y - como el Aleph de Borges, pretende - al "arrojar juntamente"- mostrar la totalidad en la unidad.

Con esta mirada, conocer el texto de Ráfagas ... es un acto de co-creación, en que el lector se dispone y se prepara desde la plataforma de sus expectativas, de tal modo que cada lectura del poemario pueda llegar a convertirse en algo tan singular como quien lo ha creado y quien lo lee.

Por eso este trabajo es sólo una e incompleta manera de interpretarlo. 


\section{CITAS}

(1) Rodrigo Eduardo Galarza pertenece a la joven generación de poetas correntinos.

Nació en el interior de la provincia, en Caá Catí, departamento de General Paz en el mes de abril de 1972. Comenzó a publicar tempranamente en diarios de Corrientes. Ejerce la corresponsalía de "Tráfico Cultural" (revista de Buenos Aires).

Es, además, fundador de un grupo literario: "Pajaro de tinta" integrado por escritores jóvenes que buscan difundir el trabajo que realizan a través de la revista del mismo nombre que Rodrigo dirige. Tiene activa participación en la SADE (seccional Corrientes)

Sus obras publicadas: Soles dormidos (poemas, 1992), Cuentionario (1er. premio del Certamen anual en narrativa de la Asociación Correntina de Cultura Inglesa), Diluvio en la memoria (poemas, 1995) y Ráfagas de pájaros (poemas, 1997. Premio Peirotén).

(2) BALAGUÉ, Miguel. Diccionario griego-español; 5a. de. Madrid, Cía. Bibliográfica Española, 1963, p. 667.

(3) BALAGUE, M.; op. cit.; pp. 672 y 133, respectivamente.

(4) CHEVALIER, J. "Introducción" (En: CHEVALIER, J. y GHEERBRANT, A. Diccionario de los símbolos. 3ra. ed.; vers. cast. M. Silvar y A. Rodríguez. Barcelona, Herder, 1991, pp. 21-22)

(5) GALARZA, Rodrigo E. Ráfagas de pájaros. Santa Fe, Macagno, 1997, p. 9.

(6) GALARZA, Rodrigo E.; op. cit.; p. 12 y 13 respectivamente.

(7) GALARZA, Rodrigo E.; op. cit.; p.15.

(8) GALARZA, Rodrigo E.; op. cit.; p.16.

(9) GALARZA, Rodrigo E.; op. cit.; p.28.

(10) GALARZA, Rodrigo E.; op. cit.; p.10.

(11) GALARZA, Rodrigo E.; op. cit.; p.13.

(12) GALARZA, Rodrigo E.; op. cit.; pp. 14 y 16, respectivamente. 
(13) GALARZA, Rodrigo E.; op. cit.; p.pp. 9 y 29, respectivamente.

(14) CHEVALIER, J. y GHEERBRANT, A.; op. cit.; p.739

(15) GALARZA, Rodrigo E.; op. cit.; p.29.

(16) GALARZA, Rodrigo E.; op. cit.; p.33. 